\title{
Improving the Resilience of Guided Transport Systems for Natural Risks
}

\author{
M. Gonzva ${ }^{1,2}$, P.-E. Gautier ${ }^{1}$, B. Barroca ${ }^{2}$, P.-A. Zitt ${ }^{3}$ and Y. Diab ${ }^{2,4}$ \\ ${ }^{1}$ Innovation Department, SYSTRA, Paris, France \\ ${ }^{2}$ Lab'Urba Laboratory \\ Paris-Est Marne-la-Vallée University, Champs-sur-Marne, France \\ ${ }^{3}$ Analysis and Applied Mathematics Laboratory \\ Paris-Est Marne-la-Vallée University, Champs-sur-Marne, France \\ ${ }^{4}$ School of Engineering of the City of Paris, France
}

\begin{abstract}
This paper provides the construction of a qualitative and systemic methodology to assess the resilience of rail transport systems against natural hazards. To illustrate the capabilities of the methodology, it is applied for the flood hazard. The resilience of rail transport systems is analysed through the failure mechanisms to which they are subjected under flood conditions. These mechanisms lead to numerous, complex failure scenarios and modelling these scenarios enables the identification the components in the system that are successively damaged as a result of a disruption. The first part presents the construction of the methodology. The second part proposes a perspective of the methodology by shifting from a qualitative to a quantitative approach by using a probabilistic framework based on Bayesian networks. The choice of Bayesian networks, the construction of the quantitative model and its capabilities are demonstrated.
\end{abstract}

Keywords: rail transport system, resilience, flood hazard, dependability methods, cascading effect, Bayesian probabilistic networks.

\section{Vulnerability of guided transport systems facing nat- ural hazards: towards a more systemic approach}

\subsection{Facts and figures about the vulnerability of guided transport system}

As many other urban infrastructures, rail transport systems are considered as vulnerable on material and functional levels to adverse weather conditions, extreme meteorological conditions and more generally, to natural hazards. Weather can affect 
operating efficiency, physical infrastructure, freight and people safety. Although this vulnerability is not theoretically demonstrated particularly due to the fact that studies investigating the effects of weather on rail transport and infrastructure are scarce [1], facts and figures on international transport systems reveal it empirically [2]. Many European projects $[3,4]$ already showed how many and complex the weather impacts on rail infrastructure and transport infrastructure are (Figure 1). In terms of rail public transport, for example, in 2003 on Netherlands rail network, among the 8000 failures recorded approximately $5 \%$ were reported to result from adverse weather conditions such as high temperatures, icing or storms [5]. Another study, based on a review of over 40000 records from the Federal Railroad Administration in the US and over the ten-year period 1995-2005, indicates a total of 861 weather related records, so less than $2.2 \%$ [6]. In this study, the most frequently reported weather phenomena causing accidents were temperature extremes and temperature variability and liquid precipitation. In terms of rail freight transport for example, the flood disaster in spring 2010 has shutdown many sections of Polish rail network; because of track damage or blocked connection to clients' railway sidings, this situation implied for the biggest private railway company in Poland the loss of contracts worth 600000 PLN, around 145000 euros. Another example in Sweden shows the vulnerability of rail freight operators to winter disruptions. Indeed, during winter 2010 the south-western Sweden was affected by unusually low temperatures and heavy snow falls over a four-month period ; the Halsberg marshalling terminal which is a centre of Sweden's rail freight operations was closed for 14 days, involving costs between 200 and 250 million SEK (between 21 and 27 million euros) [7].

Thus, a global review of literature on international incidents reveals the vulnerability of rail transport systems to natural hazards. Besides, many functional interdependencies exist between urban technical systems increasing their vulnerability; for example, the rail transport systems use electricity network to operate trains and telecommunications network for ensuring adequate levels of safety in the traffic management. But, these interactions between technical systems are often very numerous, evolving, complex and poorly known understood essentially because it goes beyond the physical boundaries of the studied system [9]. Consequently, the vulnerability of rail transport systems to natural events is not only directly due to the system itself but also to indirect effects resulting from the vulnerability of other connected technical systems. Interdependencies between technical systems generate vulnerability on these systems, at urban scale. In this context of multiple interconnections between urban technical systems, some authors refer to a "system of systems" rather than systems [10] in the context of urban risk management. For all these reasons, modern cities regard rail transport systems as critical infrastructures, generally defined as those that provides life-essential services, such as shelter, food, water, sanitation, evacuation and transportation, power and fuels, medical care, public safety, communications and access to financial resources [11]. Furthermore, two major facts require to be included in all studies dealing with the rail transport systems vulnerability. The first fact concerns the climate changes, recognized by the international scientific community, which projects that the major weather events would become more frequent and more severe in the 


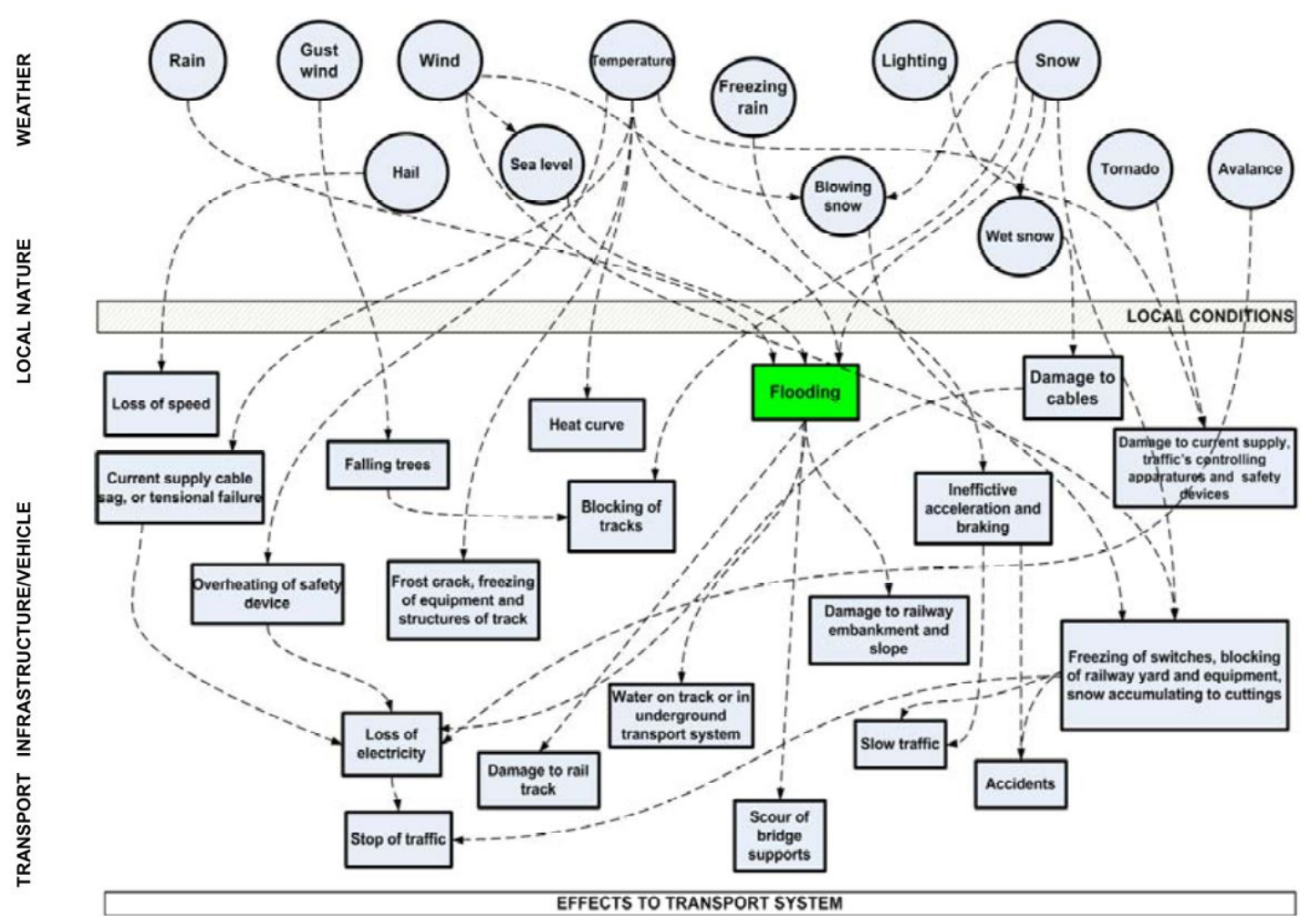

Figure 1: Conceptual map representing the multiplicity and complexity of weather phenomena impacts on rail infrastructure and transport [8]

next years. In this context, structural and organisational adaptations on rail transport systems, large and small, are essential measures to rapidly implement. The second element is about the growth of the world population; indeed, in 2014, $54 \%$ of the world's population is urban and this proportion is expected to continue to grow until $66 \%$ in 2050 [12]. This figure would have significant consequences on the current ways of operating rail transport systems, both freight and public transport systems, because of a greater need of urban and interurban mobility. In light of this situation, curbing mobility is not an option [13]. Thus, to obtain a global and integral view in the long term, the simultaneity between the growing urbanisation and the climate changes must be integrated in the management of the natural risks threaten the cities. The need of innovative, systemic and integrative methodologies is crucial and, more important, the frameworks and tools for modelling natural hazard risks should be understandable and applicable by all involved specialists, including the decision makers [14].

\subsection{A more systemic approach: the resilience concept}

Since 2000's, a new theoretical concept conveys a systemic view for analysing risks that affect cities: the resilience concept. Indeed, the concept of resilience is relevant for risks studies affecting urban technical systems because this concept provides a 
systemic approach taking into account the interactions and feedback mechanisms between the system itself, its sub-systems and components but also with elements of its environment. Due to the possibility to apply this concept to any complex system, many definitions of resilience currently exist, preventing a universal understanding of resilience [15]. Nevertheless, for urban systems, the resilience can be defined as the capacity to absorb a disturbance and to recover its functions after it [16]. Besides, the theoretical concept of resilience may be applicable taking risk management as an entry point for operationalising it [17]. An analysis of technical systems' resilience appears to be essential for converging towards urban resilience. Therefore, in order to improve the resilience of technical systems facing natural hazards, the management of risks affecting urban areas requires innovative and global methodologies integrating numerous challenges such as the complexity of systems operation, multiple internal and external interactions and environmental pressures.

This article provides the construction of a qualitative and systemic methodology to assess the resilience of rail transport systems against natural hazards. To illustrate the capabilities of the methodology, it is applied for the flood hazard. In fact, the resilience of rail transport systems is analysed through the failure mechanisms to which they are subjected under flood conditions. Indeed, these mechanisms lead to numerous, complex failure scenarios and modelling these scenarios enables to identify the components in the system that are successively damaged as a result of a disruption. A first part presents the construction of the methodology. A second part proposes a perspective of the methodology by shifting from a qualitative to a quantitative approach by using a probabilistic framework based on the Bayesian networks. The choice of Bayesian networks, the construction of the quantitative model and its capabilities are demonstrated.

\section{A global methodology for assessing resilience of rail transport systems facing flood hazards}

The global and systemic methodology proposed in this article is based on dependability concepts. Choosing to implement methods from dependability concepts for studying the resilience of rail transport systems faced by flood risks can be justified on several reasons [18]. Firstly, dependability methods are relevant for our problem inasmuch as they consist of knowing, assessing, anticipating, measuring and mastering failures in technological systems in order to limit the consequences of any such failures on human health and safety, on productivity and on the environment [19]. The second reason is that methods resulting from dependability concepts can be considered as being a wide range of methods that are specifically all adapted for the risks management [16], mainly for risks affecting urban systems. The third reason is based on the fact that recent applied research work uses these methods efficiently when modelling the way complex urban systems operate in the occurrence of a flood hazard [20]. Three methods based on dependability concepts are used to construct the 
global methodology (Figure 2) : the functional analysis, the Failure Mode and Effects Analysis (FMEA) and the causal graphs, close to the events trees. This last method provides a graphic representation of the sequence of events formed by a combination of an iterative components failures due to cascading effect.

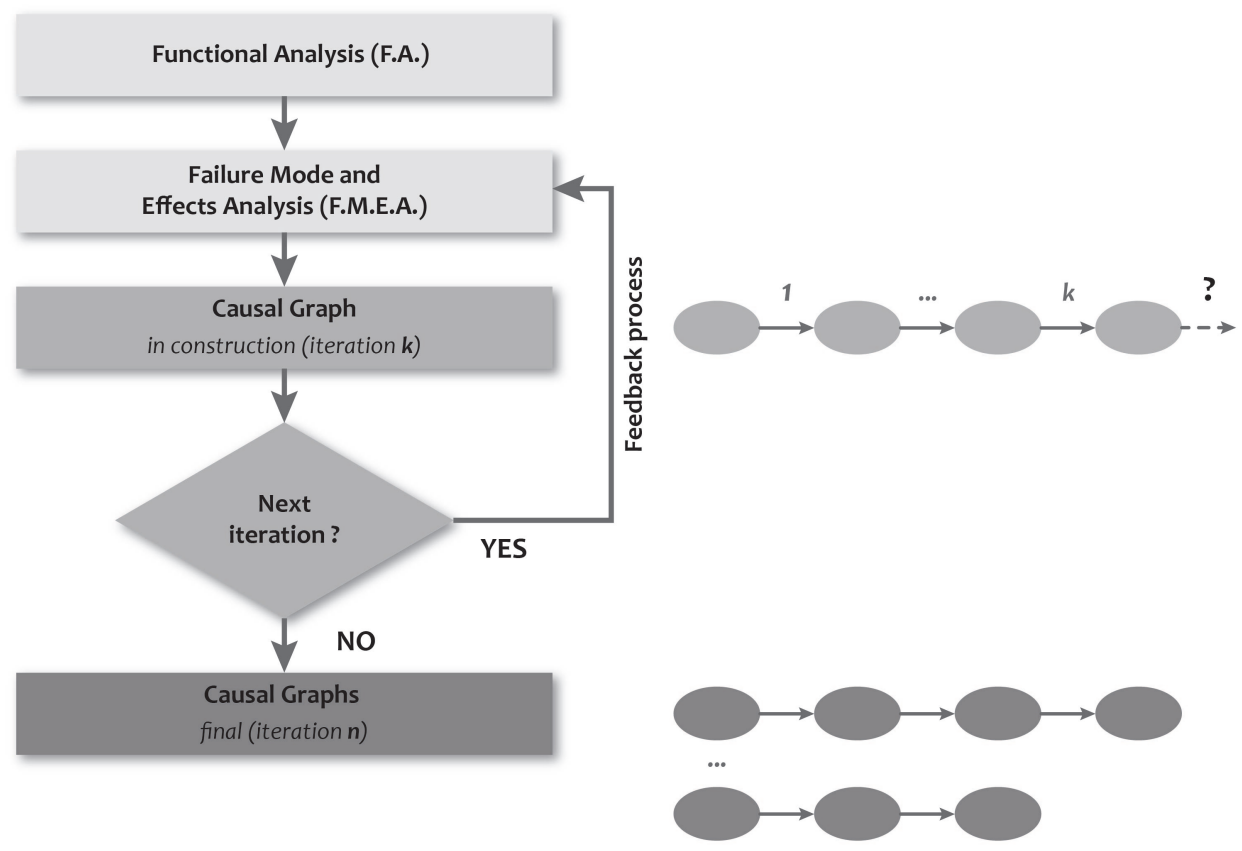

Figure 2: Methodology developed in this research (Gonzva, M.)

\subsection{Functional analysis}

The functional analysis aims at modelling the way systems operate on the basis of two mutually dependent analyses: structural analysis and functional analysis. Structural analysis defines the components in the system and provides the interactions between them in order to formulate the functions of each component in the functional analysis. Three types of interactions between subsystems are taken into consideration: contact relationships, materializing the existence of one or several physical contact between two elements; dependence relationships, indicating that the interaction from a first component to a second component is conditioned by the fact that the first component functions correctly; and, vulnerability relationships, revealing a qualitative vulnerability level to an hazard, here the flood hazard, for each element of the system. The first two interactions are related to a situation in which the system normally operates whereas the last interaction - vulnerability - is related to a crisis situation, which means during the occurrence of a flood hazard. To graphically represent the system, the Functional Block Diagram (FBD) is used [21] (Figure 3). 


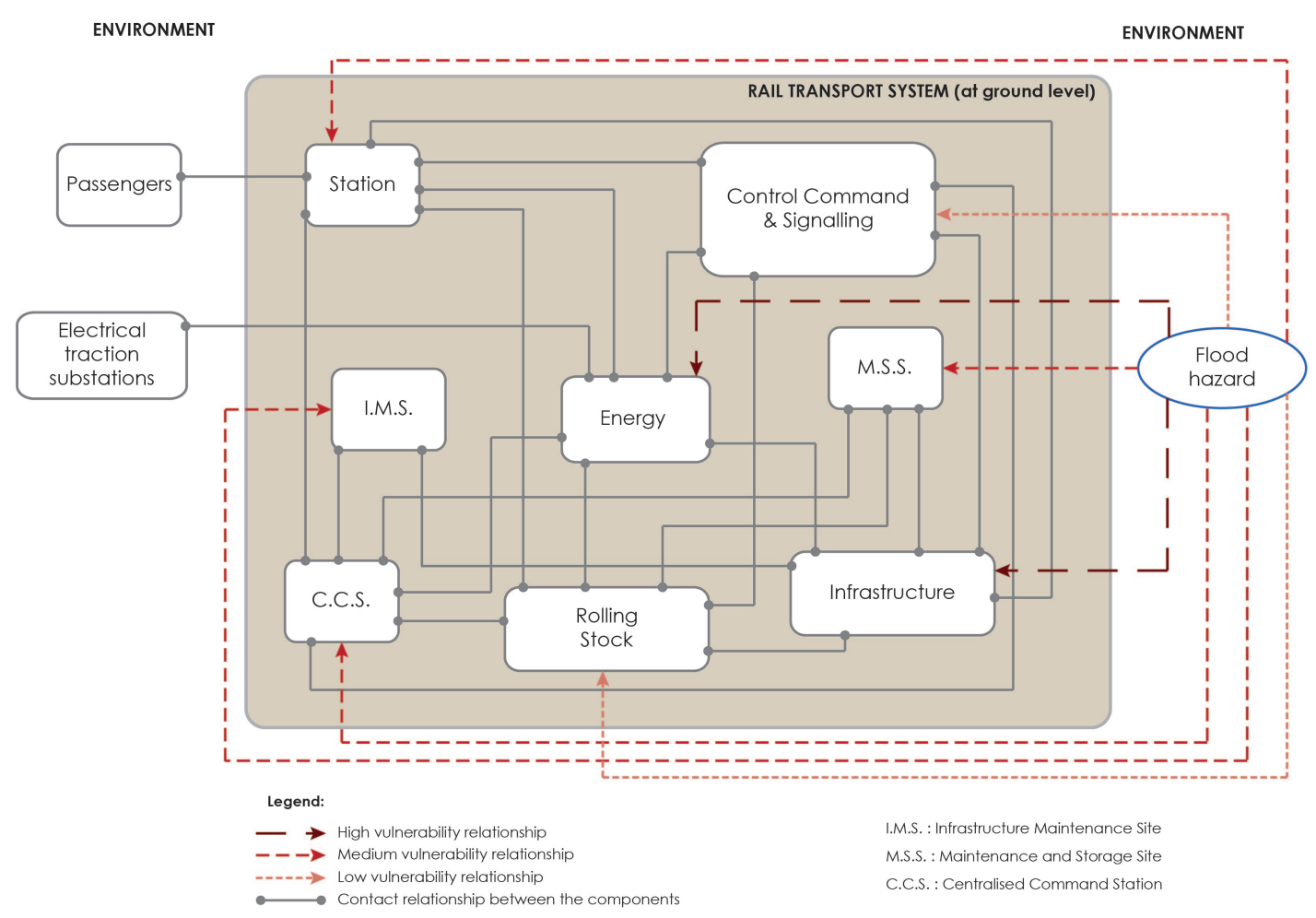

Figure 3: Functional Block Diagram (FBD) of a rail transport system in a flood hazard situation [2]

\subsection{Failure Mode and Effects Analysis}

The results of a FMEA applied on a system achieve two main objectives: identifying the critical functions of a system and, in the case of a Failure Mode and Effects and Criticality Analysis (FMECA), identifying the critical components and the causes of their failures in order to implement preventive maintenance strategies To achieve these objectives, the FMEA requires a solid knowledge of degradation mechanisms and their spread in the system [21]. The purpose in this research of the FMEA is to analyse the failure modes of all the components in the system when they are facing a hydrologic hazard. That's why, the FMEA lists for each component of each sub-system the functions provided by the component, the failure mode which here corresponds to the non-achievement of these functions due to the hydrologic hazard, the events causing the failure mode to occur and, lastly, the resulting effects of the component's dysfunction on the rest of the system. It should be noted that a FMEA may contain additional items such as the detection method(s) to notice a failure and report it, the existing corrective actions to compensate for the failure effects, or the criticity level of a failure (Figure 4). 


\begin{tabular}{|c|c|c|c|c|c|c|c|c|c|c|c|}
\hline \multicolumn{12}{|c|}{$\begin{array}{l}\text { Name of the system } \\
\text { Breakdown level of the system } \\
\text { Reference } \\
\text { Aims }\end{array}$} \\
\hline \multirow{2}{*}{$\begin{array}{c}\text { System } \\
\text { Identification } \\
\text { Number }\end{array}$} & \multirow[t]{2}{*}{ Function } & \multirow[t]{2}{*}{ Reference } & \multirow{2}{*}{$\begin{array}{l}\text { Failure } \\
\text { Mode }\end{array}$} & \multirow{2}{*}{$\begin{array}{c}\text { Failure } \\
\text { cause }\end{array}$} & \multicolumn{2}{|c|}{ Failure Effect } & \multirow{2}{*}{$\begin{array}{l}\text { Method for } \\
\text { failure } \\
\text { detection }\end{array}$} & \multirow{2}{*}{$\begin{array}{l}\text { Replacement } \\
\text { elements }\end{array}$} & \multirow{2}{*}{$\begin{array}{c}\text { Failure } \\
\text { probability }\end{array}$} & \multirow{2}{*}{$\begin{array}{l}\text { Criticity } \\
\text { level }\end{array}$} & \multirow[t]{2}{*}{ Observations } \\
\hline & & & & & $\begin{array}{l}\text { Local } \\
\text { Effect }\end{array}$ & $\begin{array}{l}\text { Final } \\
\text { Effect }\end{array}$ & & & & & \\
\hline & & & & & & & & & & & \\
\hline & & & & & & & & & & & \\
\hline
\end{tabular}

Figure 4: Template example for a FMEA (translated from [21])

\subsection{Causal graphs}

The causal graphs aim at graphically representing the successive failures of components due to cascade effect (Figure 5). Indeed, in some cases, the effect of a component failure on the system is the cause of another component failure, and so on. Thus, it is possible to obtain and represent all the chains of failures due to cascade effect by iteratively querying the FMEA to determine if the last failed component of a causal graph is not the cause of the failure of another component.

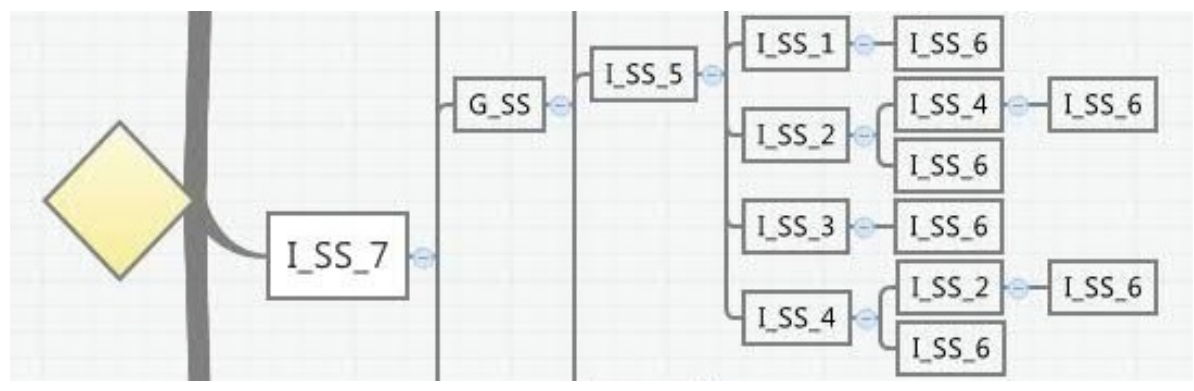

Figure 5: Extract of a chain of components failures presented as a causal graph [2])

This global methodology can be considered as powerful inasmuch as it enables to highlight the functional interdependences that exist between all the components in a rail transport system. Indeed, in the case of the occurrence of a hydrology hazard, these interdependences, inherent to the system's different elements, lead to a chain of material failures and, finally, to the overall dysfunction of the transport system.

Therefore, several conclusions can be presented at this point. Firstly, the global methodology described can be considered as innovative because, on the one hand, it is based on three methods from dependability concepts successively applied on the same system plus on an informative tool; on the other hand, the global methodology allows the assessment of the impacts of disruptions from outside the system such as a natural hazard - here, a flood hazard - which is quite different from the classical uses of these methods. Secondly, the produced chains of failures bring semi-qualitative results in terms of the vulnerability of a rail transport system facing natural hazards. Indeed, the chains of failures relatively emphasize the components of the system that 
are particularly critical for operating the system, that are particularly sensitive to a flood hazard, and that particularly spread the risk through the system via cascade effect. But, a main observation can also be raised. As described previously, the results of the methodology are semi-qualitative because the vulnerability of each component of the rail transport system is not absolutely assessed but comparatively to the vulnerability of other component. For example, a component largely presents in the chains of failures is considered as more vulnerable to flood hazard than a component scarcely presents. Thus, the question is the following: how is it possible to absolutely quantify the resilience of rail transport system's elements against natural hazards? The proposed solution in this research is building a probabilistic framework. Indeed, from the probability of a natural hazard occurring with a given intensity (a water height, for instance) through components failures due to cascade effect, the goal is to assess the probability of implement degraded modes of operation (speed restrictions of the train, for instance). Bayesian networks are therefore chosen as a relevant mathematical formalism.

\section{A probabilistic approach based on Bayesian Networks for assessing resilience of rail transport systems fac- ing flood hazards}

\subsection{Presentation of Bayesian Networks}

To overcome the limits of traditional safety and dependability risk-assessment approaches, two kinds of quantitative approaches based on the concepts of statistical inference can be employed: the frequentist approach and the Bayesian approach [22]. The frequentist approach is based on the frequency notion of probability, specifying that the probability of an event is the limit of its relative frequency of occurrence as the number of event trials becomes large, theoretically infinite. But, this approach requires a sufficiently large sample of data. The Bayesian approach, which is generally considered as more robust in situations of data scarcity [23], is concerned with the determination of the conditional probability of an unknown quantity given observations (Bensi, 2010). The use of the term "Bayesian" and the concept of conditional probability come from the Bayes' theorem, attributed to the XIX ${ }^{\text {th }}$ century mathematician and philosopher Thomas Bayes. The Bayes' theorem, describes in Equation (1) links the probability of an event based on conditions that might be related to the event.

$$
P(A \mid B)=\frac{P(A) P(B \mid A)}{P(B)}
$$

Where $P(A), P(B)$ are the probabilities of the event $\mathrm{A}$ and the event $\mathrm{B}$ without regard to each other; $P(A \mid B)$ is the conditional probability of event given that event has occurred; $P(B \mid A)$ is the conditional probability of event given that event has 
occurred. In fact, Bayes' theorem describes how the probability of event changes given information gained about the occurrence of event.

Bayesian method have given rise to a particular kind of graphical models known as Bayesian Probabilistic Networks (BPN's), facilitating the utilisation of Bayesian method for many complex problems. BPN's are acyclic directed graphs with each node representing a variable and each arc representing typically a causal relation among two variables [24], that is to say probabilistic dependencies. An example is given in Figure 6 representing a simple BPN with six variables and six causal relations between them. The probabilities calculations are based on the probabilities of the parent's nodes and the conditional probability table (CPT). A CPT is associated to each node, defining the conditional probabilities attached to the node depending of the probability distribution of its parents.

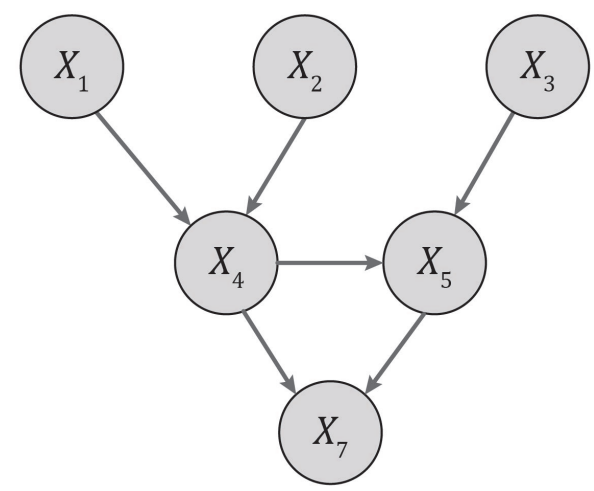

Figure 6: An example of Bayesian Probabilistic Network (Gonzva, M.)

\subsection{Advantages and potential of Bayesian Probabilistic Networks}

The Bayesian method has been chosen in this research for several reasons. Firstly, BPN's are particularly adapted for the analysis of a large variety of problems, including medical diagnosis, prognosis, epidemiology, machine diagnosis, user interfaces, natural language interpretation, planning, vision, robotics or data mining [25]. This is the case of a rail transport system facing to a natural hazard because the impacts on the components of the subsystem are multiple and complex. Secondly, due to the hierarchical structure of the system representation and the temporally and spatially varying characteristics of exposure events and consequences, the Bayesian probability theory is greatly particularly powerful for the risk assessment [26]. In a similar vein, consecutively to the global methodology described in the previous section, the BPN's are relevant inasmuch as:

- they adhere to the same hierarchical structure for the system representation : a system, divided in sub-systems, further subdivided into components ; 
- they are able to integrate the interdependences identified between components during the FMEA thanks to causal relations ;

- they have a theoretical framework similar to the causal graphs.

Thus, detailed in the next section, the construction of the BPN relative to the studied system is based on the results of the global methodology. This transition into BPN's appears opportune and quite intuitive. Thirdly, probabilistic models such as BPN's usually combine various sources of data, as existing textbooks, statistical data, databases and expert judgement. Although a particular attention is required to identify whether the sources are compatible and whether they can be combined to avoid erroneous behaviour of the model [25], this possibility to associate different sources of data allows to build powerful models. Lastly, probabilistic graphical methods such as BPN's provide a suitable framework to address problems such as failure prediction and diagnosis in a complex system $[27,28]$. This last reason gives the opportunity to quantitatively predict and diagnosis the impacts of a natural hazard on a rail transport system.

The objectives are to exploit BPN's to compute the probabilities for operating a rail transport system in degraded modes which means that the train speed is reduced, depending on the probabilities of the components failures due to a natural hazard with a given intensity.

\subsection{Constructing the Bayesian Probabilistic Network}

A BPN is generally constructed in two steps. The first step consists of defining the graphical model with the nodes and the dependence structure between all nodes. The second step consists of filling the CPTs of all BPN's nodes.

\subsubsection{Defining the graphical model}

Three categories of nodes can be identified (Figure 7):

1. A first category is natural hazard-oriented: composed of natural hazard nodes for characterizing the event, these nodes can be the height of snow, the magnitude of the earthquake or the flow rate of the water;

2. A second category is system-oriented: all the components of the system appears as nodes. These nodes are, for instance, the rail, the catenary and the ballast ;

3. A third category is operation-oriented: a unique node, depending on the components that are failed due to the impact of natural hazard, allows the characterization of the operation. This node gives the probability to operate the rail transport system at different speeds.

Besides, three categories of arcs are drawn (Figure 7): 


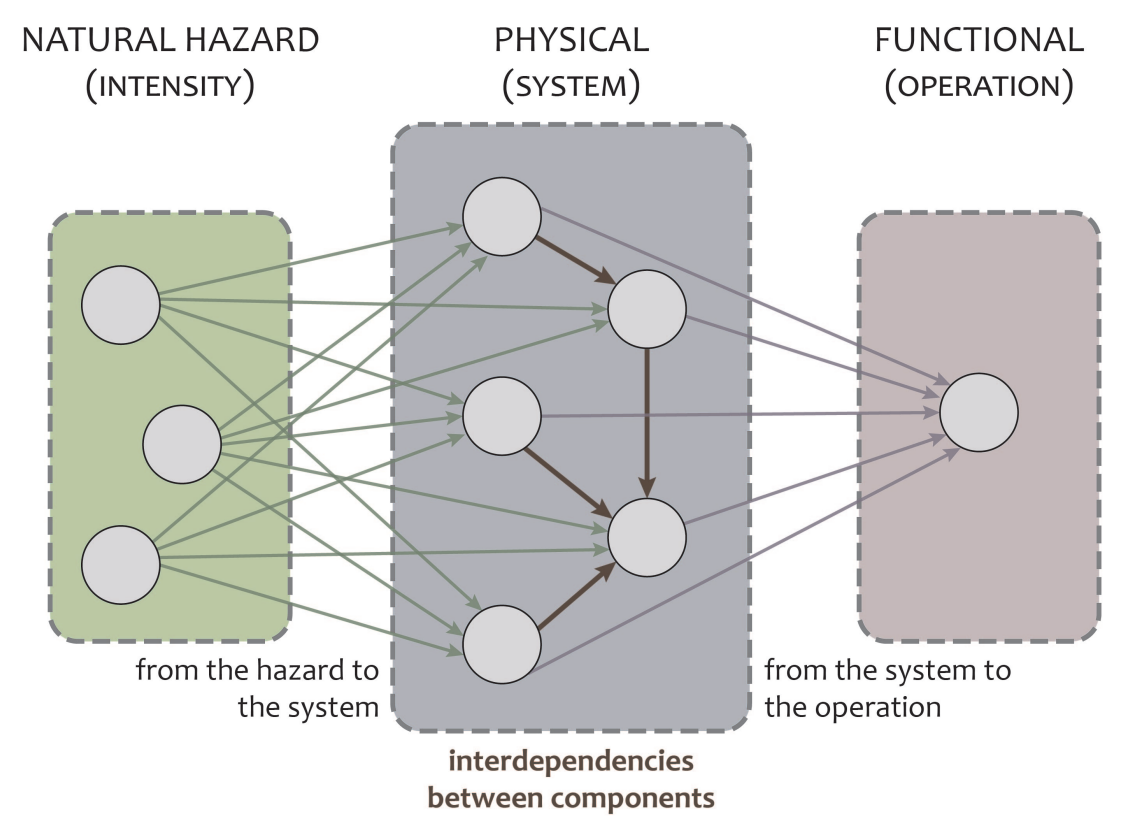

Figure 7: Principles of construction for a Bayesian Probabilistic Network dedicated to a rail transport system facing natural hazards (Gonzva, M.)

1. A first category from the natural hazard to the components: these arcs indicate that each component has a probability of being impacted by the natural hazard and, thus, a probability of being failed.

2. A second category among all components: these arcs reveal the interdependences and causal relations that exist between components. For instance, a causal relation is established between the catenary and the train: if the catenary does not supply the traction energy to the train, the train is considered to be failed because it is not able to perform its functions ;

3. A third category from all the components to a node on the system operation. According to the failure probabilities of all components, the probability of operating the trains at a given running speed is computed.

\subsubsection{Completing the conditional probability tables}

From the definition of the graphical model with nodes and causal relations, the second step consists of filling the CPTs of all nodes. CPTs of nodes can be randomly generated, but in this research, the CPTs are filled based on three kinds of sources:

- Railway standards: some data are obtained from norms governing the conception and the operation of a rail transport system to reduce the effects of flood hazards; 
- Physical models: reproducing hydrological risky phenomenon on scale models allows to deduce data, threshold values or trigger values which lead to failure components;

- Expert judgements: by interviewing experts particularly in the maintenance field and from feedbacks on past incidents due to hydrological hazards, it is possible to obtain trigger values which led to several failure components. The use of BPN's is particularly appropriate for integrating multiple data sources inasmuch as data integration is a crucial step to characterize the total material and operational impacts of natural hazards on such complex systems as rail transport.

\subsection{Application for a rail transport system and preliminary re- sults}

\subsubsection{Bayesian Probabilistic Network of a rail transport system}

BPN's can be constructed using available Bayesian softwares such as BayesiaLab ${ }^{1}$ or Netica ${ }^{2}$. In this research, Netica is used for the construction of BPN's. Indeed, it was already demonstrated, in principle, that the use of the Netica software allows for the modelling and evaluating of complex transportation infrastructure interdependencies [29].

The Figure 8 presents the Bayesian Probabilistic Network of a rail transport system in a crisis situation that is to say during the occurrence of a flood hazard.

According to the framework described in the Figure 7 :

- Two nodes define the flood hazard: "D_imm" gives the probability of the immersion time to be superior or inferior to 24 hours ; "H_eau" gives the probability that water level reaches some system components : the ballast shoulder, the sleeper, the rail foot, the rail head or the platform ;

- Eleven nodes define the main system components, with two states: not affected by the flood waters or totally submerged. Another node gives the slope of the track. Indeed, this engineering design parameter has been involved in many incidents as increasing the risk of components failures such as the ballast, in case of a flood hazard [30] this is the reason why the model include it ;

- A last node define the probability for the system to operate in degraded modes characterizing by different running speeds for trains. The implemented degraded modes depend on all states nodes impacted by the flood hazard.

\footnotetext{
${ }^{1}$ http://www.bayesialab.com

${ }^{2} \mathrm{http}: / /$ www.norsys.com/netica.html
} 


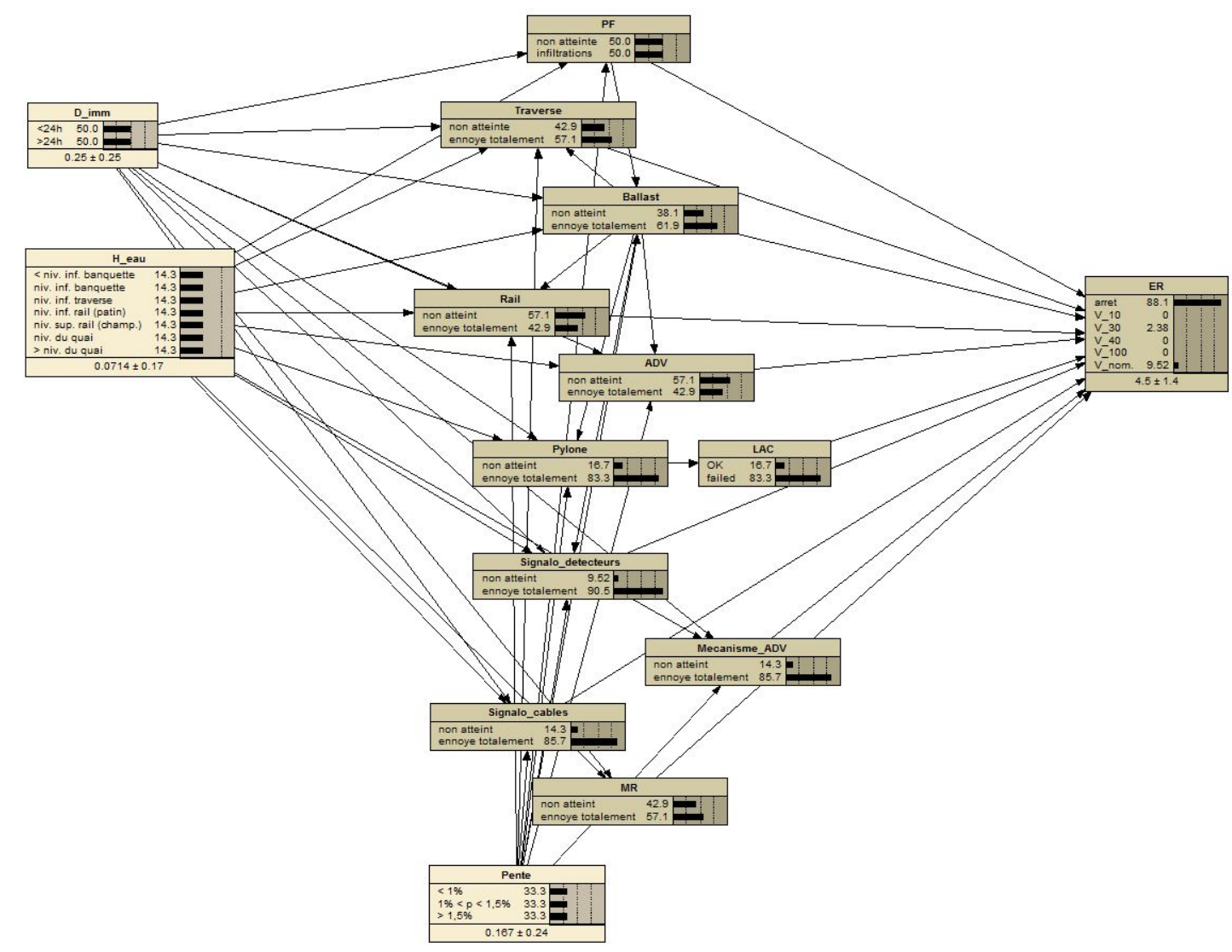

Figure 8: The Bayesian Probabilistic Network of a rail transport system

\subsubsection{Use as a diagnosis tool}

Firstly, interesting results have been found in terms of system diagnosis. Indeed, the BPN's has been used for identifying the higher failure probabilities among all components based on probabilities fixed values for the train running speeds. The objective is to determine the most critical components for a given degraded modes. In other words, from probabilities fixed values for the train running speeds, the Bayesian model provides the highest probabilities values of components failures. The Figure 9 illustrates the diagnosis capabilities of BPN's. Indeed, the probabilities values have been fixed as follows:

- the slope : about half of tracks are designed with a slope superior to $1.5 \%$, about one-quarter of tracks are designed with a slope inferior to $1 \%$ and about onequarter of tracks are designed with a slope included between $1 \%$ and $1.5 \%$;

- the degraded modes : the probability of operating the trains at nominal speed is one-third and to stop operation is two-third.

The model computes the failure probabilities of all components. It should be noted that slope values are not actual probabilities but rather designed parameters of the 
system; nevertheless, in this way, it is possible to set these values.

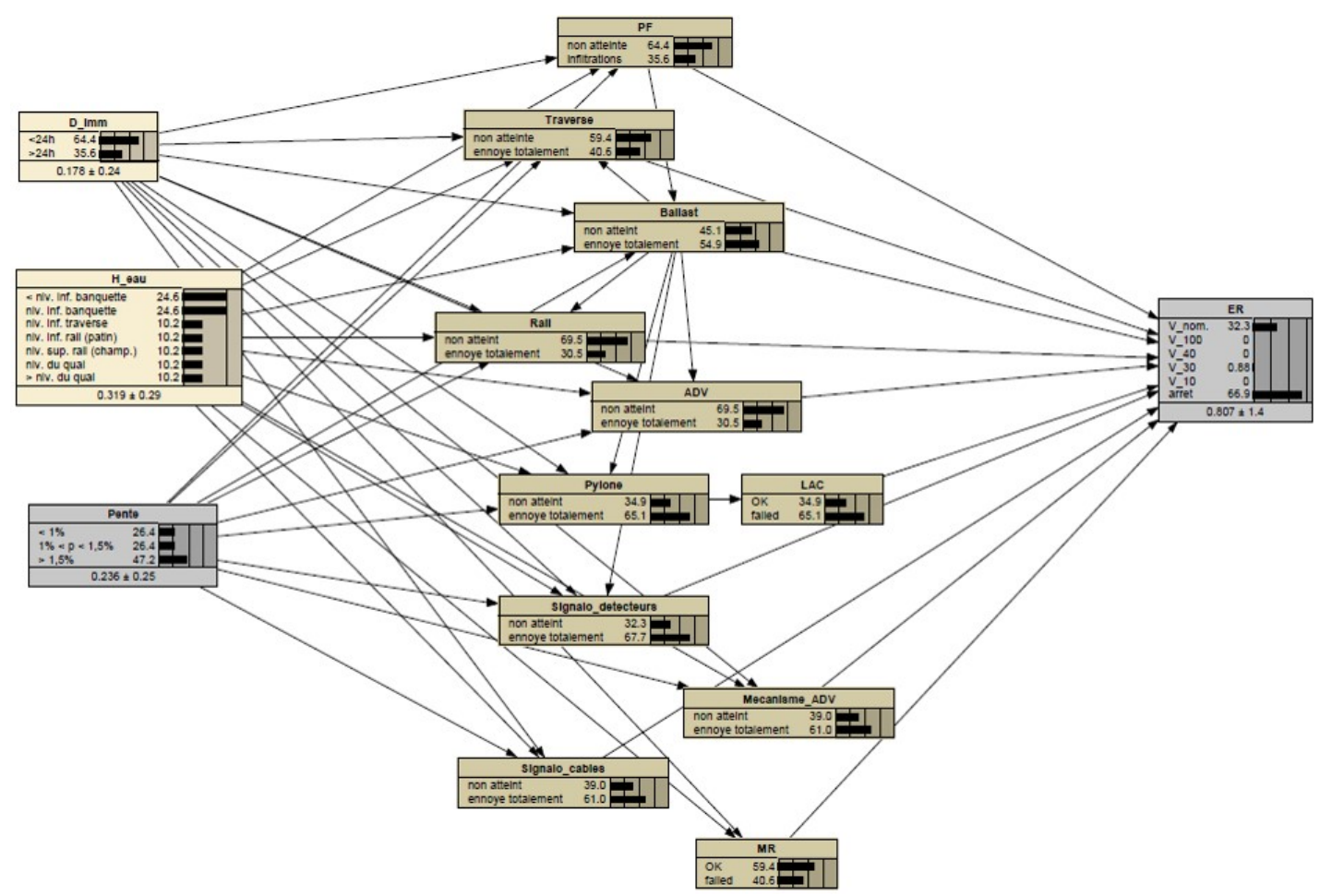

Figure 9: Use as a diagnosis tool of the Bayesian Probabilistic Network

In this example, the highest values of component failures probabilities are obtained for rail signalling components - signal cables and signal detectors. Thus, to avoid this degraded mode of operation, the model allows to identify the components to protect.

\subsubsection{Use as a prediction tool}

Secondly, interesting results have been found in terms of system prediction. The objective is to determine the degraded mode, which means the probability values for running speeds, based on probabilities fixed values of the intensity hazard. The Figure 10 illustrates the prediction capabilities of BPN's. Indeed, the probabilities values have been fixed as follows:

- the slope : about one-quarter of tracks are designed with a slope superior to $1.5 \%$, about one-quarter of tracks are designed with a slope inferior to $1 \%$ and about half of tracks of tracks are designed with a slope included between $1 \%$ and $1.5 \%$;

- the immersion time : the probability of an immersion time inferior to 24 hours is 0.9 ; 
- the water level : a probability distribution of water levels from the ballast shoulder level to the sleeper.

The model computes the probability distribution of the trains running speed. In the example, the probability value is around 0.5 for nominal speed, 0.05 for $30 \mathrm{kph}$ and 0.45 for stop operating.

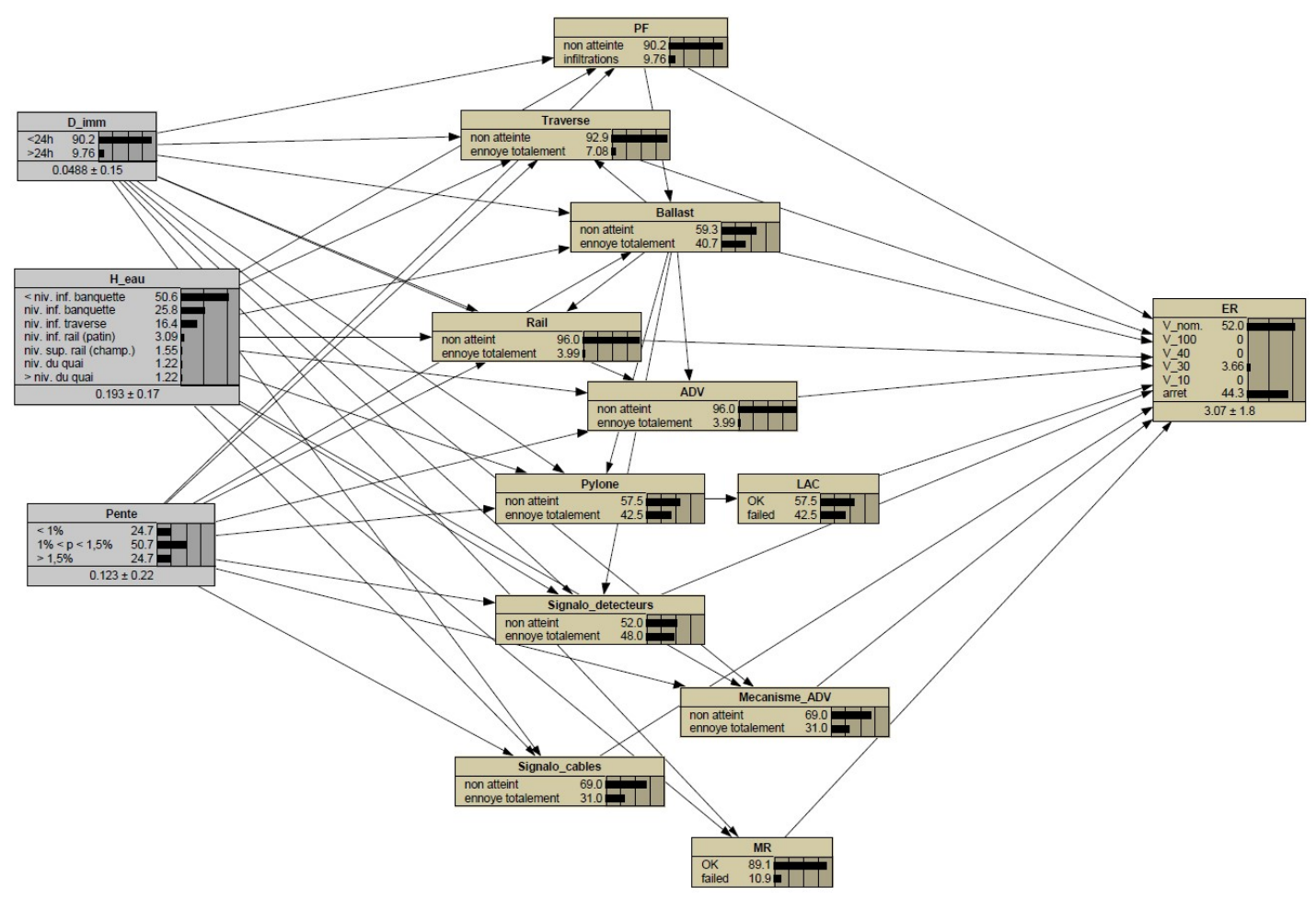

Figure 10: Use as a prediction tool of the Bayesian Probabilistic Network

\section{Conclusion}

This article provides the construction of a qualitative and systemic methodology to assess the resilience of rail transport systems against natural hazards. The methodology is applied for the flood hazard. In this research, the resilience of a rail transport system is analysed through the failure mechanisms due to cascading effect to which the components of the system are subjected under a flood hazard. The understanding and characterization of these particular types of failure mechanisms by cascading effect are crucial because they lead to numerous and complex chains of components failures. To achieve that, three methods from dependability concepts for studying the resilience have been implemented. The first method is the functional analysis. This method aims at modelling the way systems operate in two specific situations: a normal situation in which the system ordinarily operates and a crisis situation in which the system 
operates during the occurrence of a flood hazard. The second situation is particularly important because it actually provides a dysfunctional analysis of the system. The second method selected is the Failure Mode and Effects Analysis. The FMEA consists of analysing the failure modes of all the components in the system when they are facing a hydrologic hazard, the events causing the failure mode to occur and the consequential effects of the component's dysfunction on the rest of the system. The third and last method is the causal graph. Close to the event trees method, the causal graphs aim at representing the successive failures of components due to cascade effect. This method actually allows to graphically represent the results of the FMEA. Therefore, this global methodology highlights the functional interdependences that exist between all the components and that lead to a chain of components failures and, finally, to the dysfunction in terms of transport system operation.

However, this constructed methodology is specifically qualitative. To go towards a global quantitative methodology, the proposed solution is building a probabilistic framework thanks to Bayesian probabilistic networks. Some first results showed the advantages and the capabilities of this probabilistic network for diagnosis and predict the behaviour of a rail transport system facing a flood hazard. In terms of diagnosis, the model aims at determining the degraded modes, which means the probability values for implement several running speeds, based on probabilities fixed values for the intensity hazard. In terms of prediction, the objective is to characterize the degraded mode, which means the probability distribution for trains running speeds, based on probabilities fixed values of the intensity hazard. Therefore, from the probability of a natural hazard occurring with a given intensity through components failures due to cascade effect, the BPN's can compute the probability of implement degraded modes of operation - described in this research as different trains running speeds.

The next steps are multiple: validating the results based on analysis of incident feedbacks due to flood hazards, evaluating if the system breakdown is adequate or needs to be refined in some sub-elements, identifying several critical components for which failures have important consequences on system operation. This global and innovative methodology can actually contribute to improve the resilience of technical systems facing natural hazards, integrating numerous current and future challenges such as the increasing environmental pressures.

\section{Acknowledgements}

This work has been partially supported by the national project "Ville 10D - Ville d'idées" ("10D City - City of Ideas": Different Dimensions for Sustainable and Desirable Urban Development Declined in a "Top-Down" Dynamic). 


\section{References}

[1] M.J. Koetse, P. Rietveld, "The impact of climate change and weather on transport: An overview of empirical findings," Transp. Res. Part D Transp. Environ., vol. 14, no. 3, pp. 205-221, May 2009.

[2] M. Gonzva, B. Barroca, P.-E. Gautier, Y. Diab, "A modelling of disruptions cascade effect within a rail transport system facing a flood hazard," in 48th ESReDA Seminar on Critical Infrastructures Preparedness: Status of Data for Resilience Modelling, Simulation and Analysis, 2015.

[3] EWENT project (Extreme Weather impacts on transport systems) (2010-2012). Funded by the 7th framework program of the European Commission, Directorate General for Research and Technical Development. Coordinated by Technical Research Centre of Finland (VTT). http://ewent.vtt.fi

[4] MOWE-IT project (Management of Weather Events In the Transport system) (2012-2014). Funded by the 7th framework program of the European Commission. Coordinated by Technical Research Centre of Finland (VTT). http://www.mowe-it.eu

[5] A.G.P. Duinmeijer, R. Bouwknegt, Betrouwbaarheid Railinfrastructuur 2003 (Reliability Rail Infrastructure 2003). Utrecht, Prorail.

[6] B.M.A. Rossetti, "Potential Impacts of Climate Change on Railroads," in The Potential Impacts of Climate Change on Transportation Workshop, 2002, pp. $1-13$.

[7] M. Nokkala, J. Ludvigsen, R. Klaeboe, P. Leviäkangas, A.-M. Hietajärvi, S. Athanasatos, "Costs and consequences of extreme weather on European freight and logistics industries and supply chains", EWENT project D4-4, Finland, 2014.

[8] P. Leviäkangas, A. Tuominen, R. Molarius, H. Kojo, J. Schabel, S. Toivonen, J. Keränen, J. Ludvigsen, A. Vajda, H. Tuomenvirta, I. Juga, P. Nurmi, J. Rauhala, F. Rehm, T. Gerz, T. Muehlhausen, J. Schweighofer, S. Michaelides, M. Papadakis, N. Dotzek, P. Groenemeijer, EWENT project D1. Extreme weather impacts on transport systems. Finland: VTT Technical Research Centre of Finland, 2011.

[9] B. Barroca, D. Serre, "Behind The Barriers: A Resilience Conceptual Model," S.A.P.I.EN.S, vol. 6, No. April 2014, p. 11, 2013.

[10] W. Kroger, "Critical infrastructures at risk: A need for a new conceptual approach and extended analytical tools," Reliab. Eng. Syst. Saf., vol. 93, no. 12, pp. 1781-1787, Dec. 2008.

[11] J.A. McCarthy, J.P. Brashear, C. Pommerening, J.L. Siegel, J.T. Creel, T.P. Ryan, B. Stafford, L.J. Clark, B. Benson, "Critical Infrastructure Protection in the National Capital Region - Risk-Based Foundations for Resilience and Sustainability, Final Report, Volume 1," Arlington, 2005.

[12] United Nations, "World Urbanization Prospects: The 2014 Revision, Highlights (ST/ESA/SER.A/352)," New-York, 2014.

[13] European Commission, "White Paper, Roadmap to a Single European Trans- 
port Area - Towards a competitive and resource efficient transport system. $\operatorname{COM}(2011)$ 144, 28/03/2011.," 2011.

[14] D. Straub, "Natural hazards risk assessment using Bayesian networks," in Ninth International Conference on Structural Safety and Reliability (ICOSSAR 05), 2005.

[15] R. Francis, B. Bekera, "A metric and frameworks for resilience analysis of engineered and infrastructure systems," Reliab. Eng. Syst. Saf., vol. 121, pp. 90-103, 2014.

[16] S. Lhomme, "Les réseaux techniques comme vecteur de propagation des risques en milieu urbain-Une contribution théorique et pratique l'analyse de la résilience urbaine," PhD Thesis, University of Paris-Diderot, 2012.

[17] T. Mitchell, K. Harris, "Resilience: A risk management approach," London, 2012.

[18] M. Gonzva, Y. Diab, B. Barroca, P.-E. Gautier, "Résilience des systèmes de transport guidé face aux risques naturels," in Congrès Lambda-Mu 19 de Maîtrise des Risques et Sûreté de Fonctionnement, 2014, 10p.

[19] D. Noyes, F. Peres, "Analyse des systèmes - Sûreté de Fonctionnement," Techniques de l'ingénieur, 9p., 2007.

[20] M. Gonzva, M. Balsells, B. Barroca, "Mobilisation de l'analyse fonctionnelle pour l'étude de la résilience des quartiers et des systèmes de transport guidé face au risque inondation," Techniques, Sciences et Méthodes, No. 7-8, pp. 64-75, 2015.

[21] G. Zwingelstein, "La maintenance basée sur la fiabilité”, Hermes Sci. 1996.

[22] M.T. Bensi, "A Bayesian Network Methodology for Infrastructure Seismic Risk Assessment and Decision Support," PhD Thesis, University of California, Berkeley, 2010.

[23] G. Koop, Bayesian Econometrics. New-York, 2003.

[24] J. Pearl, Probabilistic Reasoning in Intelligent Systems: Networks of Plausible Inference. San Francisco, California, USA: Morgan Kaufmann, 1988.

[25] M.J. Druzdzel, F.J. Díez, "Combining knowledge from different sources in causal probabilistic models," J. Mach. Learn. Res., vol. 4, pp. 295-316, 2003.

[26] M. H. Faber, "Assessing and Managing Risks due to Natural Hazards," in ISGSR2007 First International Symposium on Geotechnical Safety and Risk, 2007, pp. 53-70.

[27] F.V. Jensen, Bayesian Networks and Decision Graphs, Springer-V. New York, 2001.

[28] R.E. Neapolitan, Learning Bayesian Networks. Pearson, 2004.

[29] M.K. Jha, R. A. Keele, Using Dynamic Bayesian Networks for Investigating the Impacts of Extreme Events. 2012.

[30] C. Pams-Capoccioni, D. Nivon, J. Amblard, G. De Cesare, T. Ghilardi, "Analysis of ballast transport in the event of overflowing of the drainage system on HSL," in RTSE - International Workshop Ballast: Issues \& Challenges, 2013, No. December. 